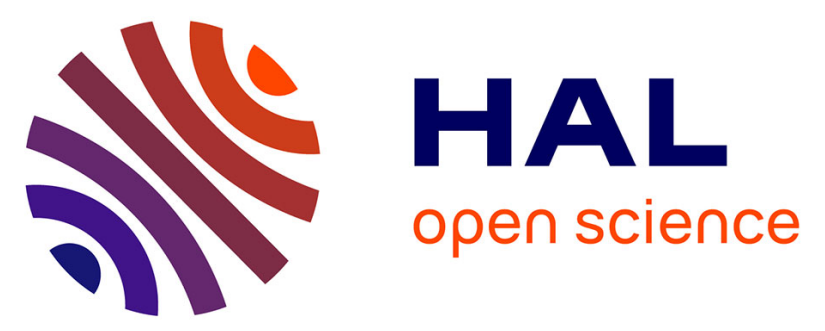

\title{
Résistance comparée de 9 espèces du genre Solanum au flétrissement bactérien (Pseudomonas solanacearum) et au nématode Meloidogyne incognita. Intérêt pour l'amélioration de l'aubergine (Solanum melongena L.) en zone tropicale humide
}

Yannick Hébert

\section{To cite this version:}

Yannick Hébert. Résistance comparée de 9 espèces du genre Solanum au flétrissement bactérien (Pseudomonas solanacearum) et au nématode Meloidogyne incognita. Intérêt pour l'amélioration de l'aubergine (Solanum melongena L.) en zone tropicale humide. Agronomie, 1985, 5 (1), pp.27-32. hal-00884729

\author{
HAL Id: hal-00884729 \\ https://hal.science/hal-00884729
}

Submitted on 1 Jan 1985

HAL is a multi-disciplinary open access archive for the deposit and dissemination of scientific research documents, whether they are published or not. The documents may come from teaching and research institutions in France or abroad, or from public or private research centers.
L'archive ouverte pluridisciplinaire HAL, est destinée au dépôt et à la diffusion de documents scientifiques de niveau recherche, publiés ou non, émanant des établissements d'enseignement et de recherche français ou étrangers, des laboratoires publics ou privés. 


\section{Résistance comparée de 9 espèces du genre Sola- num au flétrissement bactérien (Pseudomonas solanacearum) et au nématode Meloidogyne incognita. Intérêt pour l'amélioration de l'aubergine (Solanum melongena L.) en zone tropicale humide}

Yannick HÉBERT ${ }^{(1)}$

I.N.R.A., Station d'Amélioration des Plantes, Centre de Recherches des Antilles et de la Guyane, 97170 PetitBourg (Guadeloupe).

(1) Adresse actuelle : I.N.R.A. Station d'Amélioration des Plantes, 86600 Lusignan.

La bactérie Pseudomonas solanacearum, agent du flétrissement bactérien, et le nématode Meloidogyne incognita sont responsables de pertes importantes dans les cultures d'aubergine des régions tropicales humides. De par le monde, les travaux d'hybridation de l'aubergine avec des espèces apparentées sont de plus en plus nombreux. L'utilisation des croisements interspécifiques pour élargir la variabilité génétique de la résistance à ces parasites s'avère possible. La caractérisation des espèces sauvages proches de l'aubergine est une étape préliminaire.

En Guadeloupe (Antilles françaises), 9 espèces du genre Solanum, représentées par 23 populations différentes, ont été testées pour leur comportement face au flétrissement bactérien et à $M$. incognita.

Vis-à-vis de chaque parasite, nous avons décelé une grande variabilité interspécifique ainsi qu'une variabilité intraspécifique - différences de comportement entre des populations appartenant à la même espèce. La réaction à l'agent du flétrissement bactérien est la plus discriminante. Nous avons remarqué, pour nos conditions expérimentales, la résistance de certaines populations issues de $S$. aethiopicum, $S$. incanum, $S$. nigrum, $S$. torvum, $S$. viarum et $S$. warscewiezii. Le test en série pour la résistance au nématode a mis en évidence le très bon comportement de l'espèce $S$. torvum, pour les 3 populations qui la représentaient, et, à un second niveau, celui de $S$. aethiopicum.

Cette expérimentation a été peu précise. Néanmoins, les conclusions que nous obtenons sont un premier éclairage sur la variabilité disponible chez quelques espèces sauvages de Solanum non tubérifères.

Mots clés additionnels : Variabilité génétique, croisements interspécifiques. solanacearum) and root-knot nematode (Meloidogyne incognita) and possible implications in eggplant (Solanum melongena) breeding for the hot humid tropics.

The bacterium Pseudomonas solanacearum, causing bacterial wilt disease, and the root-knot nematode Meloidogyne incognita are responsible for important losses in egg-plant crops in the humid tropics. Successful crosses of egg-plant with related species have become more numerous all over the world. It may be possible to use interspecific crosses to extend the variability of genetic resistance to these pests. Classifying wild species is the next step in this direction.

Nine species of the genus Solanum, represented by 23 different populations, were tested in Guadeloupe (West Indies) in order to know their behaviour in the presence of bacterial wilt and $M$. incognita.

For each pest, there was considerable interspecific as well as intraspecific variation, i.e. behaviour differences among populations belonging to the same species. The reaction to bacterial wilt was the most discriminating. Under our conditions, resistance was specially noted in some populations of $S$. aethiopicum, $S$. incanum, $S$. nigrum, $S$. torvum, $S$. viarum and $S$. warscewiezii. Our greenhouse test showed high root-knot nematode resistance in $S$. torvum (three populations) and $S$. aethiopicum at a lower level.

Without being very precise, these results have provided preliminary ideas on the available variability in wild nontuberous Solanum species in the field.

Additional key words : Genetic variability, interspecific crosses. 


\section{INTRODUCTION}

La culture intensive de l'aubergine en régions tropicales humides pose un grave problème phytopathologique, lié à la présence de la bactérie tellurique Pseudomonas solanacearum E. Smith. Ce parasite occasionne des flétrissements particulièrement graves lorsque la culture est répétée sur des sols pauvres en calcaire et en matière organique, avec des températures supérieures à $21^{\circ} \mathrm{C}$ et une humidité atmosphérique forte (KAAN et al., 1971).

La création de nouvelles variétés, à la fois productives et suffisamment tolérantes, se heurte à une hérédité apparemment complexe du caractère de résistance et à la difficulté de plus en plus nette, pour une espèce maraîchère, d'associer une bonne valeur culturale et un objectif commercial précis (rendement, type de fruit).

Pour les Antilles françaises, nous disposons, avec la lignée pure "Ceylan SM 164 », d'un matériel adapté et, jusqu'à présent, totalement insensible à la présence de la bactérie (dans les conditions pédoclimatiques de la Guadeloupe). Mais cette variété, au fruit mauve à chair blanche, très sensible à l'anthracnose (Colletotrichum gloeosporioides Penz.), n'est pas acceptée par le marché ouest-européen et n'a, par conséquent, aucun intérêt direct. Néanmoins, elle entre comme géniteur de résistance au flétrissement bactérien dans tous les hybrides créés aux Antilles pour la culture d'exportation. Mais le contexte agricole de production met les nouvelles variétés à rude épreuve ; la valeur en combinaison des gènes de «Ceylan » ne peut faire face aux taux de contamination croissant des parcelles, fatiguées par des rotations inadaptées. De surcroît, on peut supposer l'existence d'interactions avec les attaques d'autres agents telluriques de nécrose des racines et du collet (e.g. Fusarium solani (Mart.) Sn. et H.).

La maladie n'est pas seulement importante dans les îles de la Caraïbe ; en Inde, GOPIMONY \& GEORGE (1979) en soulignent l'ampleur ainsi que la rareté des gènes de résistance. Il y a donc certainement des résultats positifs à espérer d'un élargissement de la variabilité génétique de la tolérance à $P$. solanacearum chez l'aubergine. Dans ce sens, on ne compte plus les résultats faisant état des possibilités de croisement entre l'aubergine et plusieurs espèces du même genre (GOPALKRISHNA RAO, 1980 ; KURIACHAN, 1979 ; LUDILOV, 1972 ; NASRALLAH \& HOPP, 1963 ; OMIDIJI, 1981 ; RAJASEKARAN, 1970, 1971 ; RAO, 1979 ; SHARMA et al., 1980). Sauf polyploïdie, il y a le même nombre de chromosomes et les appariements méiotiques sont le plus souvent normaux.

C'est dans le but de mieux connaître la valeur de ces espèces que nous avons expérimenté, à la Station d'Amélioration des Plantes du Centre de Recherches agronomiques des Antilles et de la Guyane (Guadeloupe), le comportement face à $P$. solanacearum de 9 espèces du genre Solanum, représentées par 23 types botaniques.

En même temps, ces espèces ont subi un test de résistance au nématode Heteroderidae, Meloidogyne incognita.

Des travaux antérieurs ont révélé la rareté des gènes de résistance connus à ces 2 agents pathogènes (FAS-
SULIOTIS, 1973). La recherche de nouvelles sources de résistances peut donc présenter, à terme, un intérêt certain.

\section{MATÉRIEL ET MÉTHODES}

\section{A. Mise en place de l'essai « flétrissement bactérien »}

Une parcelle particulièrement infestée a été utilisée, sans nécessité d'inoculation de la bactérie. Les souches pathogènes présentes et le niveau d'infestation n'étaient pas connus. Dans l'essai ont été disposés, avec les 23 numéros du tableau 1, 6 témoins utilisés en sélection ; l'un d'eux (78-21-4) est issu des récents programmes de sélection généalogique pour la résistance au flétrissement bactérien. Ce matériel, bien connu dans les conditions de la Guadeloupe, couvre presque toute la gamme des comportements vis-à-vis de $P$. solanacearum (tabl. 2).

Le dispositif comporte 5 blocs de 29 parcelles. Chacune, faite d'une seule ligne de $6 \mathrm{~m}$, compte 10 plantes. L'interligne est de $1,50 \mathrm{~m}$.

Trois semaines après le repiquage en pleine terre, nous avons dénombré les plantes de chaque parcelle. Deux notations ont eu lieu, respectivement le 16 août et le 14 septembre 1982, soit après 2 et 3 mois de végétation. Chaque parcelle a reçu une note globale égale à la proportion de plantes flétries. En général, le flétrissement ne semble pas récupérable et les plantes atteintes sont condamnées. C'est pourquoi nous n'avons pas tenu compte des degrés de flétrissement, et avons réalisé seulement une notation qualitative. Nous sommes bien conscient de la perte de précision qu'engendre ce choix.

D'autre part, nous nous sommes attaché, dans la mesure du possible (sans détruire les plantes), à distinguer les symptômes bactériens d'un flétrissement dû à d'autres causes $(F$. solani, courtilières, ...).

\section{B. Test de résistance à Meloidogyne incognita}

Le matériel testé est celui du tableau 1, sauf les populations T 3 et LS 174 de $S$. incanum et l'espèce $S$. nigrum. Dans les témoins, 78-21-4 a été remplacé par un échantillon de $S$. torvum indigène de la Guadeloupe, connu pour sa résistance aux nématodes de cette région. Les autres témoins ( $S$. melongena $\mathrm{L}$.) n'ont jamais été testés vis-à-vis de $M$. incognita.

Chaque numéro est représenté par 10 plantes cultivées en serre, sur terreau stérilisé, en pots de $17 \mathrm{~cm}$. Chaque individu (stade " 8-10 feuilles") a reçu, au contact des racines, $1 \mathrm{ml}$ d'eau contenant 1500 œufs de $M$. incognita (souche Duclos multipliée sur solanacée sensible). Après inoculation, un arrosage a permis de faire diffuser la dose dans l'ensemble du substrat de culture. La notation, $45 \mathrm{j}$ après l'inoculation (soit la durée du cycle de reproduction du nématode), s'est faite par l'attribution d'une note de 0 à 5 pour chaque plante, suivant l'importance des galles sur les racines (nombre et grosseur). 
TABLEAU 1

Espèces mises en essai pour comparaison de la résistance à $\mathrm{P}$. solanacearum.

(a) Echantillon fourni par la Station d'Amélioration des Plantes Maraîchères, I.N.R.A., Avignon.

Détermination précisée par $R$. N. LESTER (COMm. pers.).

Species tested for their resistance to $\mathrm{P}$. solanacearum.

Seed sample from the Station d'Amélioration des Plantes Maraîchères, I.N.R.A., Avignon.

Identification of species by R. N. LESTER (Pers. comm.).

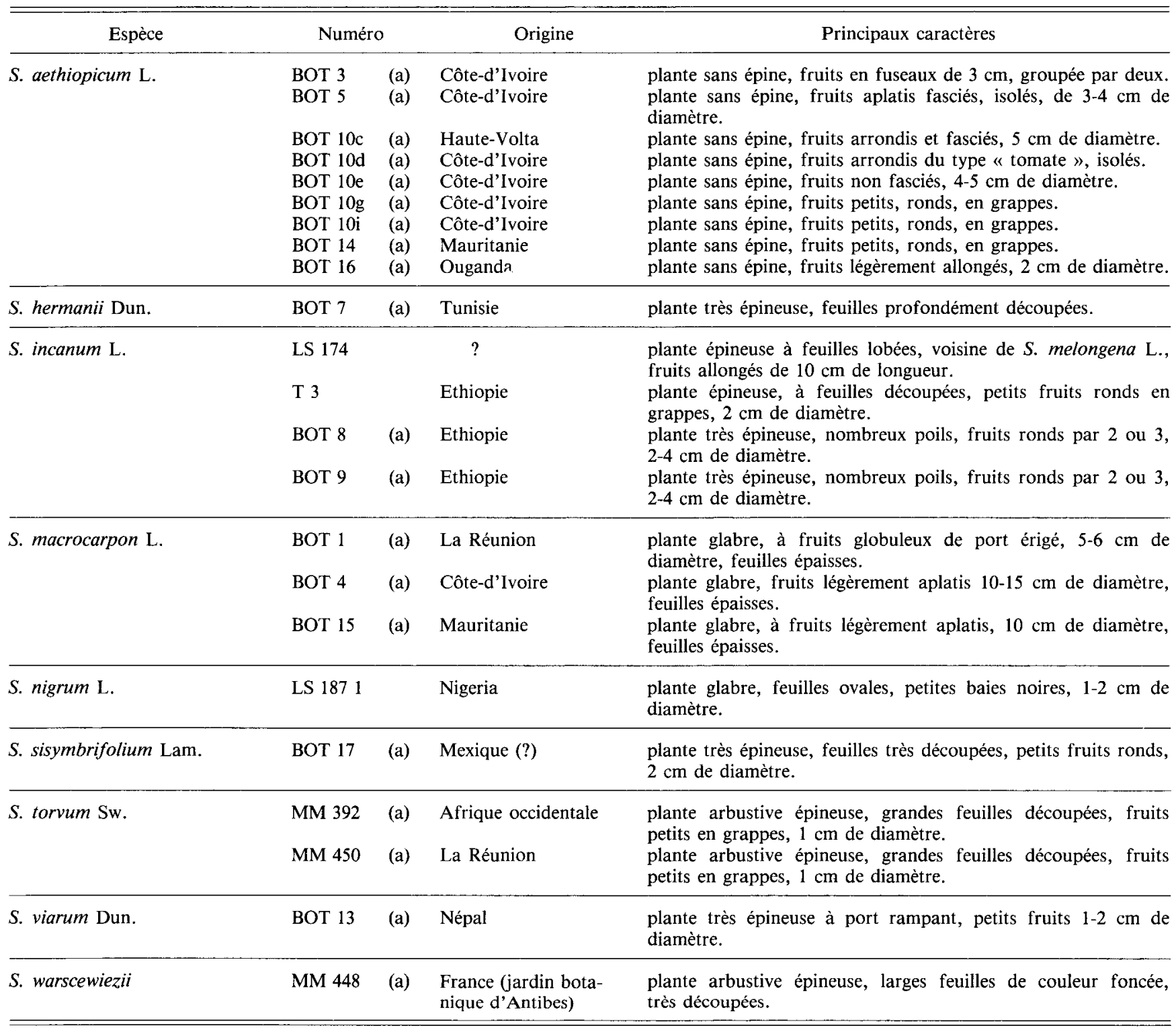

TABLEAU 2

Comportement moyen des témoins cultivés sur des sols infestés par $\mathbf{P}$. solanacearum

(conditions de la Guadeloupe, données non publiées).

Behaviour of tropically adapted eggplant varieties grown on soils infested with $\mathrm{P}$. solanacearum

(conditions of Guadeloupe, West Indies, unpublished data).

(a) $0=$ sensibilité totale (extrem susceptibility)

$5=$ résistance totale (total resistance)

\begin{tabular}{|c|c|}
\hline Variété & Résistance relative (a) \\
\hline Zébrina & 1 \\
\hline Kalenda F1 & $3-4$ \\
\hline Ceylan & 5 \\
\hline Aranguez & 1 \\
\hline L 17 & 4 \\
\hline $78-21-4$ & 4 \\
\hline
\end{tabular}

\section{Analyses statistiques}

Pour les 2 expériences, nous avons procédé à l'analyse de variance. Les proportions issues de la notation "flétrissement bactérien " ont subi la transformation angulaire modifiée (DAGNÉLIE, 1978) afin de stabiliser les variances et de satisfaire aux exigences de normalité de la méthode d'analyse.

Cette adaptation a permis d'arriver à des variables satisfaisantes : les effets résiduels n'étaient pas corrélés aux valeurs estimées $(r=0,0000)$ et leur distribution s'est avérée quasiment normale : symétrie parfaite, coefficient d'aplatissement un peu élevé, après test des coefficients de Pearson. Mais l'analyse de variance et le test des comparaisons multiples de moyennes de NEWMAN \& KEULS sont assez robustes 
dans ces conditions limites aussi bien en ce qui concerne le risque de $1^{\text {re }}$ espèce que pour la puissance. Ceci a été confirmé par un test non paramétrique de FRIEDMAN (CONOVER, 1971) dans le cas de l'essai «flétrissement » (cf. § III.A).

Les comparaisons de moyennes ont été réalisées par la méthode de NEWMAN-KeUls (KeULS, 1952 ; NEWMAN, 1939), plus conservative que l'analyse de variance (test F). La discrimination entre populations a été ainsi plus sûre.

\section{RÉSULTATS ET DISCUSSION}

Dans l'ensemble, ces expérimentations se caractérisent par une faible précision, due au mauvais contrôle des inoculations - particulièrement pour $P$. solanacearum -, à la variabilité d'expression des agents pathogènes et à un fort effet " milieu ». Néanmoins, les différences interspécifiques sont telles qu'elles se manifestent tout de même, avec parfois une signification élevée.

\section{A. Résistance au flétrissement bactérien}

Les dégâts observés sont assez importants : sur l'ensemble des parcelles, 25 p. 100 des plantes sont atteintes à la $1^{\text {re }}$ date ( 2 mois après repiquage) ; le flétrissement affecte 45 p. 100 des plantes à la $2^{e}$ date ( 3 mois après repiquage). Dans les 2 cas, les différences globales entre nos 29 populations sont hautement significatives $(P<0,01)$. Le test non paramétrique de FRIEDMAN (CONOVER, 1971) appliqué à ces données conduit aux mêmes conclusions, son résultat étant très hautement significatif $(\mathrm{P}<0,001)$.

A la $1^{\text {re }}$ notation, la méthode de comparaison des moyennes de populations au risque 5 p. 100 permet de dégager un groupe de 10 génotypes sauvages et de 3 témoins (tabl. 3). Il s'agit d'un ensemble d'espèces ayant présenté moins de 10 p. 100 de plantes flétries, 30 p. 100 après calcul de l'intervalle de confiance. Les 3 témoins «Ceylan », «78-21-4 » et « L 17 » ont extériorisé leur bonne résistance, et « Zébrina » a confirmé sa sensibilité. Nous retrouvons les résultats du tableau 2. "Kalenda », pour sa part, a beaucoup

TABLEAU 3

Différences interspécifiques pour la résistance au flétrissement bactérien.

(a) Abréviation du nom d'espèce suivie du numéro de référence au tableau 1.

(b) Nombre total de plantes observées.

(c) Pourcentage de plantes flétries.

(d) Exclu de l'analyse en raison d'un trop faible effectif total.

Interspecific differences in bacterial wilt resistance.

(a) Abbreviation of species name followed by reference number, see table 1.

(b) Total number of plants.

(c) Percentage of wilted plants.

(d) Excluded from the analysis on account of an insufficient number of plants.

\begin{tabular}{|c|c|c|c|c|c|c|c|}
\hline \multicolumn{4}{|c|}{ Date 1} & \multicolumn{4}{|c|}{ Date 2} \\
\hline Espèce (a) & Effectif (b) & $\%(c)$ & $\begin{array}{c}\text { Test de } \\
\text { NewMan-Keuls } \\
\alpha=0,05\end{array}$ & Espèce (a) & Effectif (b) & $\%$ (c) & $\begin{array}{c}\text { Test de } \\
\text { Newman-Keuls } \\
\alpha=0,05\end{array}$ \\
\hline Inc. BOT 9 & 37 & 80 & & Aet. BOT 3 & 43 & 96 & \\
\hline Zébrina & 47 & 77 & & Aet. BOT 9 & 37 & 96 & \\
\hline Aet. BOT 14 & 46 & 74 & & Aet. BOT14 & 46 & 96 & \\
\hline Her. BOT 7 & 42 & 70 & & Aet. BOT 5 & 34 & 96 & \\
\hline Mac. BOT 1 & 42 & 60 & & Mac. BOT 4 & 43 & 95 & \\
\hline Inc. BOT 8 & 26 & 50 & & Inc. BOT 8 & 26 & 95 & \\
\hline Mac. BOT 15 & 36 & 38 & & Her. BOT 7 & 42 & 93 & \\
\hline Aet. BOT 3 & 43 & 38 & & Mac. BOT 1 & 42 & 93 & \\
\hline Aet. BOT 5 & 34 & 34 & & Zébrina & 47 & 92 & \\
\hline Mac. BOT 4 & 43 & 34 & & Mac. BOT 15 & 36 & 90 & \\
\hline Aet. BOT $10 \mathrm{i}$ & 46 & 31 & & Aet. BOT $10 \mathrm{i}$ & 46 & 76 & \\
\hline Sis. BOT 17 & 38 & 28 & & Nig. LS 187-1 & 38 & 57 & \\
\hline Kalenda & 48 & 23 & & Sis. BOT 17 & 38 & 55 & \\
\hline Inc. T 3 & 26 & 11 & & Kalenda & 48 & 48 & | \\
\hline Nig. LS $187-1$ & 38 & 8 & & War. MM 448 & 11 & 31 & \\
\hline (Aranguez) (d) & (7) & (6) & & (Aranguez) (d) & (7) & (30) & \\
\hline Ceylan & 50 & 6 & & Inc. T 3 & 26 & 25 & \\
\hline War. MM 448 & 11 & 4 & & Aet. BOT $10 \mathrm{c}$ & 48 & 9 & \\
\hline Tor. MM 392 & 34 & 4 & & $78-21-4$ & 25 & 6 & \\
\hline L 17 & 50 & 4 & & L 17 & 50 & 6 & \\
\hline $78-21-4$ & 25 & 4 & & Ceylan & 50 & 6 & \\
\hline Inc. LS 174 & 29 & 3 & & Tor. MM 392 & 34 & 4 & \\
\hline Aet. BOT $10 \mathrm{~g}$ & 41 & 3 & & Inc. LS 174 & 29 & 3 & \\
\hline Via. BOT 13 & 44 & 3 & & Aet. BOT $10 \mathrm{~g}$ & 41 & 3 & \\
\hline Aet. BOT $10 \mathrm{e}$ & 46 & 3 & & Via. BOT 13 & 44 & 3 & \\
\hline Aet. BOT $10 \mathrm{c}$ & 48 & 3 & & Aet. BOT $10 \mathrm{e}$ & 46 & 3 & \\
\hline Tor. MM 450 & 49 & 2 & & Aet. BOT 16 & 37 & 2 & \\
\hline Aet. BOT $10 \mathrm{~d}$ & 50 & 2 & & Tor. MM 450 & 49 & 2 & \\
\hline Aet. BOT 16 & 37 & 2 & & Aet. BOT $10 \mathrm{~d}$ & 50 & 2 & \\
\hline
\end{tabular}


souffert puisqu'on ne peut la distinguer des populations ayant flétri à $50 \mathrm{p}$. 100 . Ceci confirme certains aspects de son comportement en grande culture, lorsqu'elle est installée sur sol très contaminé.

La $2^{\mathrm{e}}$ notation a été plus discriminante, montrant que les différences entre sensibles et résistants se sont accentuées par rapport à la variation intraparcellaire. Dix populations ont subi moins de 25 p. 100 de pertes, et, parmi elles, 8 n'ont montré de symptômes qu'à moins de 6 p. 100. Tous ces génotypes se trouvaient dans le groupe des résistants à la $1^{\text {re }}$ notation. Inversement, on notera que les plus mauvais étaient des plantes déjà fortement atteintes. Il n’y a donc eu aucune "récupération" de la part des lignées déjà malades. Ceci explique que nos 2 mesures soient bien corrélées $(r=0,90 \mathrm{P}<0,001)$. Parmi les témoins notons que "Kalenda " accuse une sensibilité plus forte. «Ceylan », «L 17 » et « 78-21-4 », pour leur part, confirment leur résistance (tabl. 3).

Au moins sur les 3 premiers mois de végétation, ces résultats corroborent ceux obtenus par certains auteurs indiens et japonais : GOPIMONY \& GEORGE (1979) et SRINIVASAN et al. (1969) font état de la rareté des gènes de résistance chez l'aubergine, et de la totale immunité de la forme sauvage $S$. insanum, sous-espèce proche de $S$. incanum. MochIZUKI \& YAMAKAWA (1979a) mettent l'accent sur la résistance de $S$. integrifolium, $S$. torvum et $S$. maroniense; MOCHIZUKI \& YAMAKAWA (1979b) rapportent, en outre, la résistance de $S$. xanthocarpon Schrad. \& Wendl. (=S. viarum Dun.), avec quelques nuances.

\section{B. Résistance à $M$. incognita}

Les différences entre nos 26 familles se sont avérées hautement significatives $(P<0,01)$. Néanmoins, le test de NeWMan-Keuls au seuil de risque 5 p. 100 ne montre pas un partitionnement très net de notre échelle de comportements, ceci en raison de son caractère plutôt conservatif (tabl. 4).

Contrairement aux résultats de FASSULIOTIS (1973), l'espèce $S$. sisymbrifolium n'est que moyennement résistante (note 2,5). Par contre, remarquons la totale résistance de $S$. torvum (MM 392 et MM 450). Notre témoin local de l'espèce s'est avéré toutefois légèrement sensible (4 plantes et 5 plantes sur 9 ont reçu respectivement les notes 1 et 0 ).

L'espèce $S$. aethiopicum a montré de l'hétérogénéité entre les différents numéros qui la représentaient: BOT 10i a justifié la valeur moyenne 0,8 alors que BOT $10 \mathrm{~g}$ a été noté 4,2 .

Les témoins utilisés ont manifesté une résistance intermédiaire, avec les notes suivantes :

\begin{tabular}{ll} 
Ceylan & 1,6 \\
L 17 & 1,6 \\
Zébrina & 2,2 \\
Aranguez & 2,3 \\
Kalenda & 1,3 \\
\hline Moyenne générale & 1,9
\end{tabular}

Ces valeurs sont statistiquement semblables et régulièrement réparties autour de la moyenne de l'essai.
TABLEAU 4

Différences interspécifiques dans la résistance à $\mathrm{M}$. incognita. (a) (b) Voir légendes tableau 3.

Interspecific differences in the resistance to $\mathrm{M}$. incognita. (a) (b) See references in table 3.

\begin{tabular}{lccc|c}
\hline \hline \multicolumn{1}{c}{ Espèce (a) } & Effectif (b) & Note & $\begin{array}{c}\text { Test de } \\
\text { NewMAN-Keuls } \\
\alpha=0,05\end{array}$ \\
\hline Tor. MM 392 & & & & \\
Tor. MM 450 & 10 & 0,0 & \\
Tor. Guadeloupe & 10 & 0,0 & \\
Aet. BOT 10i & 9 & 0,5 & \\
Aet. BOT 3 & 10 & 0,8 & \\
Aet. BOT 14 & 10 & 1,1 & \\
Kalenda & 10 & 1,2 & \\
Mac. BOT 4 & 10 & 1,3 & \\
Ceylan & 10 & 1,4 & \\
L 17 & 10 & 1,6 & \\
Aet. BOT 10e & 10 & 1,6 & \\
Mac. BOT 15 & 10 & 1,6 & \\
War. MM 448 & 10 & 1,8 & \\
Inc. BOT 8 & 9 & 1,9 & \\
Aet. BOT 5 & 9 & 2,0 & \\
Inc. BOT 9 & 10 & 2,0 & \\
Mac. BOT 1 & 10 & 2,1 & \\
Zébrina & 9 & 2,2 & \\
Aranguez & 10 & 2,2 & \\
Sis. BOT 17 & 10 & 2,3 & \\
Aet. BOT 16 & 10 & 2,5 & \\
Her. BOT 7 & 10 & 2,5 & \\
Via. BOT 13 & 10 & 2,5 & \\
Aet. BOT 10c & 10 & 2,5 & \\
Aet. BOT 10d & 10 & 3,3 & \\
Aet. BOT 10g & 10 & 3,3 & \\
& 9 & 4,2 & \\
\hline \hline
\end{tabular}

Cette homogénéité de comportement pourrait s'expliquer par l'absence de sélection dans ce matériel pour la résistance aux nématodes à moins que des gènes de résistance n'y existent pas au départ.

La précision d'un tel essai devra être améliorée, surtout par l'observation d'effectifs plus importants. Néanmoins, notre expérience fait ressortir des populations intéressantes :

S. torvum : MM 392, MM 450, Guadeloupe

S. aethiopicum : BOT 10i, BOT 3, BOT 14.

\section{CONCLUSION}

Deux essais, réalisés sur 9 espèces de Solanum, ont montré l'existence d'une variabilité interspécifique et intraspécifique - entre populations d'une même espèce - pour le comportement en présence de $P$. solanacearum et $M$. incognita. Des différences particulièrement importantes ont été observées à propos de la résistance au flétrissement bactérien.

Nous retiendrons le bon comportement des populations :

S. aethiopicum : BOT 10c, 10d, 10e, 10g, 16
S. incanum
: LS 174
S. torvum : MM 392, 450
S. viarum : BOT 13
S. warscewiezii : MM 448.

Sur les lignes les plus marquées par le flétrissement, nous avons pu observer une forte variabilité. Il est possible que, compte tenu de lá conduite de l'essai 
(niveau d'infestation, notation), les conditions aient été limites pour l'expression de la résistance, entrâ̂nant une plus forte variation phénotypique. Néanmoins, il existe une variabilité interspécifique. Il reste à la confirmer par des essais plus précis, portant sur un plus grand nombre de plantes, réalisés sur un « terrain » épidémiologique mieux connu (substrat stérilisé avant infection artificielle). Cette remarque mise à part, nous insisterons sur l'intérêt que peuvent présenter les 6 espèces citées ci-dessus pour l'amélioration de la résistance de l'aubergine au flétrissement bactérien. Outre l'utilisation des espèces résistantes comme porte-greffes (BEYRIES, 1979 ; MOCHIZUKI \& YAMAKAWA, 1979a), celles-ci pourraient constituer la source d'une nouvelle variabilité, faite d'allèles ou de gènes inconnus chez l'aubergine. Certains auteurs ont déjà fait ressortir l'intérêt de ces espèces dans la mesure où ils ont noté une résistance totale des hybrides aubergine $\times$ Solanum sauvages (RAO, 1980 ; SRIVIVASAN et al., 1969) et l'on sait que de nombreux croisements interspécifiques sont possibles et débouchent sur des hybrides partiellement fertiles (LAKSHMI et al., 1981 ; NASRALLAH \& HOPP, 1963 ; RAO, 1980).

Le test de la résistance à $M$. incognita a été moins puissant face à l'expression de la variabilité. Certaines différences se sont présentées, de façon assez nette.
Nous avons retenu pour leur bon comportement les espèces et numéros :

S. aethiopicum : BOT 3, 10i, 14

\section{S. torvum : MM 392, 450, Guadeloupe.}

Cette étude est une étape préliminaire dans l'ensemble des travaux, encore peu avancés, d'exploration des espèces apparentées à l'aubergine. Si l'utilisation des croisements interspécifiques s'avère propre à fournir un progrès génétique suffisant, ce travail devra être repris dans des conditions plus favorables à une meilleure précision : connaissance des agents pathogènes - $P$. solanacearum surtout - contrôle de la variabilité environnementale et intraspécifique.

Reçu le 14 septembre 1983. Accepté le 16 juillet 1984.

\section{REMERCIEMENTS}

Nous voulons remercier ici Marie-Christine DAUNAY (Station d'Amélioration des Plantes Maraîchères, I.N.R.A. Avignon) et Kunio YamaKawa (Vegetable and Ornamental Crops Research Station - Japon) qui ont bien voulu nous fournir les échantillons de la collection de Solanum.

Nous tenons à remercier également José ANAïs (Station de Zoologie - I.N.R.A. Guadeloupe) pour sa collaboration à la mise en place de l'essai-nématodes.

\section{RÉFÉRENCES BIBLIOGRAPHIQUES}

Beyries A., 1979. Le greffage, moyen de lutte contre les parasites telluriques des Solanées cultivées pour leurs fruits. Thèse Doct., Université des Sciences et Techniques du Languedoc, $166 \mathrm{p}$.

Conover W. J., 1971. Practical non-parametric statistics, John Wiley \& sons, Inc. New York, 462 p.

Dagnélie P., 1978. Théorie et méthodes statistiques. Tome 2. Les Presses Agronomiques de Gembloux, Belgique, $463 \mathrm{p}$.

Fassuliotis G., 1973. Susceptibility of eggplant, Solanum melongena, to root-knot nematode, Meloidogyne incognita. Plant Dis. Rep., 57 (7), 606-608.

Gopalkrishna Rao M., 1980. A note on an interspecific hybrid between Solanum melongena $\times$ S. torvum. Curr. Res., 9 (7), 117-118.

Gopimony R., George M. K., 1979. Screening brinjal varieties for wilt resistance. Agric. Res. J. of Kerala, 17 (1), 7-10.

Kaan F., Pecaut P., Beramis M., 1971. La sélection des Solanées pour la résistance au flétrissement bactérien. Journ. Int. Etude Product. maraîchères, organisées par la Fondation Ford, Ibadan, Nigeria, 21-25/06/1971.

Keuls M., 1952. The use of the studentized range in connection with an analysis of variance. Euphytica, 1, 112-122.

Kuriachan P., 1979. Cytogenetic studies on Solanum macrocarpon L. $\times$ S. melongena L. hybrid and its colchiploid. Ind. J. Bot., 2 (2), 159-165.

Lakshmi V. V. S., Moorty K. V., Rao B. G. S., 1981. Studies on the incompatibility among spinous Solanum species. III. The degree on divergence of $S$. incanum $\mathrm{L}$. and $S$. melongena $\mathrm{L}$. in relation to other solanums. Incompatibility Newsletter, 13, 101-103.

Ludilov V. A., 1972. Possibilité de croisement de Solanum melongena et $S$. integrifolium Poir. (en russe). S-kh. Biol., 7 (5), 782-785.

Mochizuki H., Yamakawa K., 1979a. Potential utilization of bacterial wilt resistant Solanum species as rootstock for commercial eggplant production. Bull. Veg. Ornam. Crops Res. Stn. (Japan), 6, 11-18 (en japonais), in Hortic. Abstr., 51, 1257.
Mochizuki H., Yamakawa K., 1979b. Resistance of selected eggplant cultivars and related wild Solanum species to bacterial wilt (Pseudomonas solanacearum). Bull Veg. Ornam. Crops Res. Stn. (Japan) 6, 1-10 (en japonais). Abstr. in Rev. Plant Pathol., 61, 2045 .

Nasrallah M. E., Hopp R. J., 1963. Interspecific crosses between Solanum melongena L. (eggplant) and related Solanum species. Proc. Am. Soc. Hortic. Sci., 83, 571-574.

Newman D., 1939. The distribution of range in samples from a normal population expressed in terms of an independent estimate of standard deviation. Biometrika, 31, 20-30.

Omidiji M. O., 1981. Cytogenetic studies of the F1 hybrid between the African eggplant Solanum gilo Raddi and Solanum melongena L., Hortic. Res., 21, 75-82.

Rajasekaran S., 1970. Cytogenetic studies of the F1 hybrid (Solanum indicum L. $\times S$. melongena L.) and its amphiploid. Euphytica, 19 (2), 217-224.

Rajasekaran S., 1971. Cytological studies on the F1 hybrid (Sola num xanthocarpum Schrad. \& Wendl. $\times S$. melongena L.) and its amplidiploid. Caryologia, 24, 261-267.

Rao M. G., 1980. A note on an interspecific hybrid between Solanum melongena and S. torvum. Curr. Res., 9 (7), 117-118.

Rao N. N., 1979. The barriers to hybridization between Solanum melongena and some other species of Solanum. In Hawkes J. G., Lester R. N., Skelding A. D. : "The biology and Taxonomy of the Solanaceae ». Academic Press, London.

Sharma D. R., Chowdhury J. B., Ahuja Uma, Dhankhar B. S. 1980. Interspecific hybridization in genus Solanum. A cross between $S$. melongena and $S$. khasianum through embryo culture. Z. Pflanzenzücht., 85, 248-253.

Srinivasan K., Copimony R., Swaminathan M., Pillai P. K., 1969 On the resistance of a wild Brinjal variety to bacterial wilt. Agric. Res. J. of Kerala, 7, 39-40, in Hortic. Abstr., 40 (4), 8544. 